\title{
Is it Possible to Modulate Microbiota in the Perinatal Period?
}

\section{Mariella Baldassarre $^{1 *}$, Nicola Laforgia ${ }^{1}$, Alfredo Miccheli ${ }^{3}$ and Paola Mastromarino ${ }^{2}$}

${ }^{1}$ Dipartimento di Scienze Biomediche ed Oncologia-Neonatology and NICU Section, University of Bari, Italy

${ }^{2}$ Dipartimento di Sanità Pubblica e Malattie Infettive, Italy

${ }^{3}$ Dipartimento di Chimica, "La Sapienza" University, Roma, Italy

The influence of intestinal flora on human health is continuous from birth to old age. The first contact with the bacteria during and immediately after birth has an important role in the development of the intestinal flora and then on the development of mucosal immune system of the newborn [1]. Intestinal microbiota of the newborn is related to his susceptibility to infection and sensibility to antigen's environmental issues in the early years of life [1]. The correct sequence in the microbial species that colonize the intestine of the newborn in the first period of life leads to immune and metabolic programming that has a long term influence on the risk of diseases [2]. For example, birth by cesarean section appears to increase the risk of celiac disease, type 1 diabetes and asthma, which are generally associated with excessive or aberrant $\mathrm{T}$ helper responses [3]. It has been suggested that contact between mother and fetus microbiota may occur already in the uterus. Detectable amounts of bacteria or bacterial DNA were detected in the placenta as well as in healthy membranes and amniotic fluid and this microbial contact, although not yet fully understood, is associated with a systemic immune response in the newborn. The presence of microbial DNA in the placenta could significantly modulate innate immunity in the 'fetal gut [3]. The ability to modulate the development and the composition of the intestinal microbiota in order to improve the health of both mother and child is the basis of the use of probiotics. The administration of probiotics to the mother in the perinatal period results in changes in intestinal microbiota of women that are reflected in changes of immune factors and in microbiota of the newborn. The assumption of VSL \# 3, a mixture of lactobacilli and bifidobacteria in the last month of pregnancy and during the first month of lactation resulted in a significant increase in breast milk concentrations of secretory IgA and anti-inflammatory and immunomodulatory cytokines such as TGF- $\beta$ and IL-10 [4]. Furthermore, the concentration of lactobacilli and bifidobacteria in the intestinal microbiota of infants breastfed by mothers who took the probiotic, was higher compared with infants of control mothers [4]. In agreement, the consumption of probiotics by Finnish mothers before birth and during lactation induced modulation of colonization and growth of bifidobacteria in the gut of their children, in particular by increasing the diversity of species of bifidobacteria [5]. In addition, the administration to mothers during the last weeks of pregnancy of probiotics containing a strain of lactobacilli resulted in an increased amount of B. longum fecal in infants [6] and has proven effective in protecting the child from atopic dermatitis [7].

A recent study [8] showed that oral intake of VSL \# 3 in the last trimester of pregnancy is associated with a modulation of the vaginal microbiota and secretion of cytokines, with significantly higher levels of IL-4 and IL-10, with an anti-inflammatory effect, and reduction of Eotaxin, a pro-inflammatory chemokine. The probiotic treatment was associated with a stabilization of vaginal immune responses and a global anti-inflammatory effect on the vaginal community.

These changes in vaginal microbiota could have important implications in the prevention of vaginal infections and therefore of preterm birth.

In conclusion, probiotics given to the mother during pregnancy and lactation may be a valuable means for modulating the microbiota of the mother and the newborn.

\section{References}

1. Sjogren YM, Tomicic S, Lundberg A, Böttcher MF, Björkstén B, et al. (2009) Influence of early gut microbiota on the maturation of childhood mucosal and systemic immune responses. Clin Exp Allergy 39: 1842-1851.

2. Nicholson JK, Holmes E, Kinross J, BurcelinR, Gibson G, et al. (2012) Host-Gut microbiota metabolic interactions. Science 336: 1262-1267.

3. Rautava S, Luoto R, Salminen S, Isolauri E (2012) Microbial contact during pregnancy, intestinal colonization and human disease. Nat Rev Gastroenterol Hepatol 9: 565-576.

4. Baldassarre MEF, Cacciotti A, Miccheli F, Urbano NL, Mastromarino P (2012) VSL\#3 probiotic maternal supplementation affects breast milk composition and newborn faecesmicrobiota. Digestive and Liver Disease 44: S253.

5. Gueimonde M, Sakata S, Kalliomäki M, Isolauri E, Benno Y, et al. (2006) Effect of maternal consumption of lactobacillus GG on transfer and establishment of fecal bifidobacterialmicrobiota in neonates. J Pediatr Gastroenterol Nutr 42 166-170.

6. Lahtinen SJ, Boyle RJ, Kivivuori S, Oppedisano F, Smith KR, et al. (2009) Prenatal probiotic administration can influence Bifidobacteriummicrobiota development in infants at high risk of allergy. J Allergy ClinImmunol 123: 499501.

7. Rautava S, Kainonen E, Salminen S, Isolauri E (2012) Maternal probiotic supplementation during pregnancy and breast-feeding reduces the risk of eczema in the infant. J Allergy Clinlmmunol 130: 1355-1360.

8. Vitali B, Cruciani F, Baldassarre ME, Capursi T, Spisni E, et al. (2012) Dietary supplementation with probiotics during late pregnancy: outcome on vaginal microbiota and cytokine secretion. BMC Microbiol 12: 236.
*Corresponding author: Mariella Baldassarre, Dipartimento di Scienze Biomediche ed Oncologia-Neonatology and NICU Section, University of Bari, Italy, Tel: +393296114818; E-mail: mariellabaldassarre@gmail.com

Received March 15, 2014; Accepted March 19, 2014; Published March 21, 2014

Citation: Baldassarre M, Laforgia N, Miccheli A, Mastromarino P (2014) is it Possible to Modulate Microbiota in the Perinatal Period? J Clin Case Rep 4: e133. doi:10.4172/2165-7920.1000e133

Copyright: (C) 2014 Baldassarre M, et al. This is an open-access article distributed under the terms of the Creative Commons Attribution License, which permits unrestricted use, distribution, and reproduction in any medium, provided the original author and source are credited. 